\title{
POTENCIAL EROSIVO DE PASTILHAS E BALAS DURAS “ZERO AÇÚCAR" DISSOLVIDAS EM ÁGUA E SALIVA ARTIFICIAL.
}

\author{
Marcos Rodrigo RITA ${ }^{1}$ \\ E-mail:marcosritasjb@hotmail.com. \\ Maria Mercês Aquino Gouveia FARIAS² \\ E-mail:mercesfarias@gmail.com. \\ Eliane Garcia da SILVEIRA ${ }^{3}$ \\ E-mail: elianesilveira@univali.br
}

\section{RESUMO}

O consumo de balas ácidas está associado à etiologia da erosão dental. Esta pesquisa objetivou avaliar o potencial erosivo de pastilhas e balas duras "zero açúcar" disponíveis comercialmente. Foram analisadas as balas: Halls ${ }^{\oplus}$ mini (extraforte, melancia, mentol e cereja) e as pastilhas Melagrião ${ }^{\oplus}$ (limão) e Valda ${ }^{\circledast}$ friends (mentol). Constituíram-se 2 grupos: balas e pastilhas dissolvidas em água duplamente deionizada (G-1) e balas e pastilhas dissolvidas em saliva artificial (G-2). O pH foi mensurado utilizando-se um potenciômetro e eletrodo combinado de vidro previamente calibrado com soluções padrão $\mathrm{pH} 7,0$ e pH 4,0, antes de

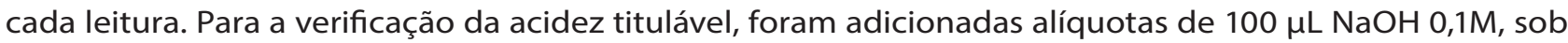
agitação constante até alcançar pH 7,0. Os resultados foram submetidos à Análise de Variância (ANOVA) e as comparações das médias realizadas pelo teste Tukey, em um nível de $5 \%$ de significância $(p<0,05)$. As balas e pastilhas dissolvidas em água (G-1) apresentaram valores de pH inferiores a 5,5, com exceção do sabor mentol Valda ${ }^{\oplus}(\mathrm{pH}=6,1)$, que diferiu significantemente dos demais. Após diluição em saliva artificial (G-2) todos os sabores apresentaram valores de pH superiores a 5,5. Na comparação entre os grupos (G-1 e G-2), observou-se elevação significativa do pH no grupo G-2. Observou-se redução significativa da acidez titulável após diluição na saliva artificial (G-2). Conclui-se que a maioria das balas e pastilhas analisadas são ácidas, mas diferem quanto ao seu potencial erosivo. A saliva artificial atuou elevando o $\mathrm{pH}$ e reduzindo a acidez titulável.

DESCRITORES: EROSÃO DENTÁRIA; COMPORTAMENTO ALIMENTAR; DOCES; CONCENTRAÇÃO DE ÍONS DE HIDROGÊNIO; ACIDEZ

Acadêmico, Bolsista de Iniciação Científica do Curso de Odontologia da Universidade do Vale de Itajaí (UNIVALI), Itajaí/SC, Brasil.

2 Mestre em Odontopediatria, Professora da Disciplina de Clínica Integrada Infantil do Curso de Graduação em Odontologia da Universidade do Vale de Itajaí (UNIVALI), Itajaí/ SC, Brasil.

3 Mestre em Odontopediatria, Professora da Disciplina de Clínica Integrada Infantil do Curso de Graduação em Odontologia da Universidade do Vale de Itajaí (UNIVALI), Itajaí/ SC, Brasil. 
ISSN 1983-5183

\title{
EROSIVE POTENTIAL OF "ZERO SUGAR" HARD CANDIES AND TABLETS DISSOLVED IN WATER AND ARTIFICIAL SALIVA.
}

\begin{abstract}
The consumption of acid candy is associated with the etiology of dental erosion. This research aimed to evaluate the erosive potential of commercially available "zero sugar" tablets and hard candy. The "zero sugar" Halls ${ }^{\circledR}$ mini hard candies (extra-strong, watermelon, menthol and cherry) and the Melagrião ${ }^{\circledR}$ (lemon) and Valda ${ }^{\circledR}$ friends (menthol) tablets were analyzed. Two groups were formed: candies and tablets dissolved in distilled water (G-1) and candies and tablets dissolved in artificial saliva (G-2). The pH was measured using a potentiometer and combined glass electrode previously calibrated with standard solutions $\mathrm{pH} 7.0$ and $\mathrm{pH} 4.0$ before each reading. For verification of the titratable acidity, aliquots of $100 \mu \mathrm{L} 0.1 \mathrm{M} \mathrm{NaOH}$ were added under constant stirring until the $\mathrm{pH}$ reached 7.0. The results were submitted to Analysis of Variance (ANOVA) and Tukey test comparisons, at a level of $5 \%$ of significance $(p<0.05)$. The candies and tablets dilution in water (G-1) had $\mathrm{pH}$ values below 5.5, except for menthol flavor Valda ${ }^{\circledR}(\mathrm{pH}=6.1)$, which differed significantly from the others. After dilution in artificial saliva (G-2) all flavors had $\mathrm{pH}$ values above 5.5 . In the comparison between the groups (G-1 and G-2), a significant elevation of pH was observed in the G-2 group. After dilution in water, the Melagrião ${ }^{\circledR}$ lemon and Mini Halls ${ }^{\circledast}$ watermelons had the highest titratable acidity differing significantly from the others. It was observed a significant reduction of titratable acidity after dilution in the artificial saliva (G-2). According to the results, most of the candies and tablets analyzed are acidic. Dilution in artificial saliva raised the $\mathrm{pH}$ and reduced the titratable acidity, thereby reducing the erosive potential of the products.
\end{abstract}

DESCRIPTORS: TOOTH EROSION; FEEDING BEHAVIOR; DULCES; HYDROGEN-ION CONCENTRATION; ACIDITY.

\section{INTRODUÇÃO}

A erosão dental é o resultado do desgaste químico dos tecidos dentários duros provocados por ácidos de origem intrínseca, extrínseca e/ou substâncias quelantes, sem o envolvimento bacteriano 1,2. Os ácidos intrínsecos provêm de vômitos crônicos decorrentes de desordens alimentares e refluxo gastresofágico, ocasionando o contato do suco gástrico com os dentes. Os de origem extrínseca são oriundos do meio ambiente ( $\mathrm{pH}$ ácido de piscinas, gases industriais corrosivos), dieta (alimentos e bebidas ácidas), medicamentos (ácido ascórbico, ácido acetil salicílico, anti-histamínicos) e drogas², 2, 4 .

Dentre os ácidos de origem extrínseca, os presentes na dieta são considerados os mais relevantes na etiologia da erosão dental. Estudos sobre a prevalência da erosão dental e fatores de risco, apontaram o consumo frequente de doces/balas ácidas como um potencial fator de risco para desgaste erosivo $0^{5,6,7,8}$.

Produtos livres de açúcar são rotulados como seguros para os dentes, por não apresentam cariogenicidade. Entretanto, o fato de serem livres de açúcar não os isentam de poder provocar danos à estrutura dentária, pois podem conter ácidos na sua composição (ácido cítrico, fosfórico, ascórbico, málico, tartárico, oxálicio, carbônico, ácido fumárico), e dessa forma, estarem relacionados à etiologia da erosão dental $\left.\right|^{9,10}$.

A complexa etiologia da erosão dental envolve a participação de fatores físico-químicos, como: tipo de ácido, pH, acidez titulável, potencial quelante, concentração de cálcio e fosfato, temperatura e adesividade ${ }^{11,}$ 
RITA MR, FARIAS MMAG, SILVEIRA EG. Potencial erosivo de pastilhas e balas duras "zero açúcar" dissolvidas em água e saliva artificial. Rev. Odontol. Univ. Cid. São Paulo 2018 jul/set 30(3) 246-55

ISSN 1983-5183

12. Dentre esses fatores destacam-se o pH, acidez titulável e conteúdo de cálcio, como os parâmetros mais relevantes para mensurar o potencial erosivo de um produto"1.

Por outro lado, a erosão dental é modulada por fatores biológicos; entre eles destaca-se a saliva que apresenta propriedades biológicas que protegem os tecidos dentários duros do desgaste erosivo, através de suas propriedades como: capacidade tampão, concentração de cálcio, fosfato, flúor, fluxo e limpeza ${ }^{4,13,}$ 14,15 .

O conhecimento do potencial erosivo e do papel protetor da saliva são significantes para a compreensão da dinâmica da erosão dental. Dessa forma, o objetivo desta pesquisa foi mensurar o potencial erosivo de pastilhas e balas duras "zero açúcar" dissolvidas em água e saliva artificial.

\section{MATERIAIS E MÉTODOS}

Foram selecionadas balas duras mini Halls ${ }^{\circledR}$ e as pastilhas Melagrião ${ }^{\circledR}$ e Valda ${ }^{\circledR}$ disponíveis comercialmente, todas tendo como característica em comum a disponibilização de várias pastilhas contidas em uma mesma embalagem (Quadro 1). As balas e pastilhas foram distribuídas em 2 grupos:

G-1- balas e pastilhas dissolvidas em água destilada;

G-2 -balas e pastilhas dissolvidas em saliva artificial.

Quadro 1- Sabores selecionados e composição do produto segundo o fabricante.

\begin{tabular}{|c|c|}
\hline $\begin{array}{l}\text { Sabor/ } \\
\text { Marca }\end{array}$ & Composição segundo fabricante \\
\hline $\begin{array}{l}\text { Halls }^{\circledR} \text { Mini } \\
\text { Extra Forte }\end{array}$ & $\begin{array}{l}\text { Isomalte, aspartame e acesulfame de potássio, aromatizantes, acidulante ácido cítrico, espes- } \\
\text { sante carboximetilcelulose sódica. }\end{array}$ \\
\hline $\begin{array}{l}\text { Halls }^{\circledR} \text { Mini } \\
\text { Melancia }\end{array}$ & $\begin{array}{l}\text { Isomalte, aspartame e acesulfame de potássio, aromatizantes, acidulante ácido cítrico, espes- } \\
\text { sante carboximetilcelulose sódica. Corante vermelho } 40 .\end{array}$ \\
\hline $\begin{array}{l}\text { Halls }^{\circledR} \text { Mini } \\
\text { Mentol }\end{array}$ & $\begin{array}{l}\text { Isomalte, aspartame e acesulfame de potássio, aromatizantes, acidulante ácido cítrico, espes- } \\
\text { sante carboximetilcelulose sódica. Corante azul brilhante. }\end{array}$ \\
\hline $\begin{array}{l}\text { Halls }^{\circledR} \text { Mini } \\
\text { Cereja }\end{array}$ & $\begin{array}{l}\text { Isomalte, aspartame e acesulfame de potássio, aromatizantes, acidulante ácido cítrico, espes- } \\
\text { sante carboximetilcelulose sódica. Corante vermelho } 40 \text { e indigotina. }\end{array}$ \\
\hline $\begin{array}{l}\text { Valda }{ }^{\circledR} \\
\text { Friends }\end{array}$ & $\begin{array}{l}\text { Sorbitol, sucralose, aromatizantes, antiaglutinante estearato de magnésio, corante artificial } \\
\text { azul brilhante. }\end{array}$ \\
\hline $\begin{array}{l}\text { Melagrião }{ }^{\circledR} \mathrm{C} \\
\text { Limão }\end{array}$ & $\begin{array}{l}\text { Isomalte, ácido ascórbico, acesulfame K, acidulante: ácido cítrico, mentol, aroma idêntico ao } \\
\text { natural de limão, aroma idêntico ao substituto de mentol, aroma idêntico ao natural de gengi- } \\
\text { bre e corante azul brilhante. }\end{array}$ \\
\hline
\end{tabular}

Fonte: Fabricante.

\section{ANÁLISE DO PH E ACIDEZ TITULÁVEL}

Para cada um dos sabores selecionados foram utilizadas 6 embalagens. As balas presentes em cada embalagem foram trituradas com um pistilo e gral de porcelana $305 \mathrm{~mL}$ (Nalgon Equipamentos Científicos. Itupeva - SP). Do pó resultante, pesaram-se 2 amostras de $20 \mathrm{~g}$ gramas, utilizando-se, para esse fim, uma balança eletrônica analítica e de precisão (AE200S Mettler-Toledo Ind. e Com. Ltda. Alphaville, 
ISSN 1983-5183

Barueri-SP-Brasil). As amostras de pó foram dissolvidas em $125 \mathrm{~mL}$ de água destilada (G-1) e $125 \mathrm{~mL}$ de saliva artificial (20mM NaHCO, $\left.3 \mathrm{mM} \mathrm{NaH}_{2} \mathrm{PO}_{4} \mathrm{H}_{2} \mathrm{O} 1 \mathrm{mM} \mathrm{CaCl}_{2} 2 \mathrm{H}_{2} \mathrm{O}\right)(\mathrm{G}-2)$ com o auxílio de um bastão de vidro até se obter uma solução homogênea. Destas soluções, obtiveram-se 3 amostras de $30 \mathrm{~mL}$ para cada um dos sabores presentes em cada grupo. Esse processo permitiu a leitura em triplicata do pH e acidez titulável ${ }^{16}$.

$\mathrm{O}$ pH foi mensurado sob temperatura ambiente e agitação constante (Agitador Magnético Fisaton), utilizando-se um potenciômetro e eletrodo combinado de vidro (Tec-2 Tecnal) previamente calibrado com soluções padrão pH 7,0 e pH 4,0, antes de cada leitura.

Para mensuração da acidez titulável adicionaram-se alíquotas de $100 \mu \mathrm{L} \mathrm{NaOH} \mathrm{0,1} \mathrm{M,} \mathrm{sob} \mathrm{agitação}$ constante (Agitador Magnético Fisaton), até o pH alcançar 7,0. Esse procedimento foi realizado para cada um dos sabores.

Os resultados foram submetidos à análise estatística através da Análise de Variância (ANOVA). As comparações das médias realizadas pelo teste Tukey em um nível de $5 \%$ de significância $(p<0,05)$

\section{RESULTADOS}

As balas e pastilhas dissolvidas em água (G-1) apresentaram valores de $\mathrm{pH}$ inferiores a 5,5, com exceção do sabor mentol Valda ${ }^{\circledR}(\mathrm{pH}=6,1)$, que diferiu significantemente dos demais. Após diluição em saliva artificial (G-2) todos os sabores apresentaram valores de $\mathrm{pH}$ superiores a 5,5. Na comparação entre os grupos (G-1 e G-2), observou-se elevação significativa do pH no grupo G-2 (Tabela 1).

Tabela 1. Média e desvio-padrão dos valores de $\mathrm{pH}$ de diferentes sabores de balas duras, após diluição em água (G-1) e saliva artificial (G-2).

\begin{tabular}{llll}
\hline Sabores & Marca comercial & G-1 (água) & G-2 (saliva artificial) \\
\hline Cereja & Mini Halls ${ }^{\oplus}$ & $3,9 \pm 0,05 \mathrm{bB}$ & $8,1 \pm 0,04 \mathrm{aA}$ \\
Extraforte & Mini Halls $^{\oplus}$ & $3,2 \pm 0,07 \mathrm{bcB}$ & $6,8 \pm 0,03 \mathrm{bcA}$ \\
Mentol & Mini Halls & $3,0 \pm 0,02 \mathrm{cB}$ & $6,8 \pm 0,08 \mathrm{bcA}$ \\
Melancia & Mini Halls $^{\oplus}$ & $2,9 \pm 0,03 \mathrm{cB}$ & $6,6 \pm 0,02 \mathrm{cA}$ \\
Mentol & Valda $^{\circledR}$ & $6,1 \pm 0,07 \mathrm{aB}$ & $7,4 \pm 0,02 \mathrm{bA}$ \\
Limão & Melagrião $^{\oplus}$ & $2,8 \pm 0,04 \mathrm{cB}$ & $6,6 \pm 0,02 \mathrm{CA}$ \\
\hline \hline
\end{tabular}

Médias seguidas da mesma letra minúscula na vertical e maiúscula na horizontal não diferem significativamente entre si pelo teste de Tukey $(p<0,05)$.

Em relação à acidez titulável observou-se que, após diluição em água (G-1), os sabores limão Melagrião ${ }^{\circledR}$ e melancia Mini Halls ${ }^{\circledast}$ apresentaram a mais elevada acidez tittulável, diferindo significantemente dos demais. Na comparação entre os grupos (G-1 e G-2) houve redução significativa da acidez titulável após diluição na saliva artificial (G-2) (Tabela -2). 
RITA MR, FARIAS MMAG, SILVEIRA EG. Potencial erosivo de pastilhas e balas duras "zero açúcar" dissolvidas em água e saliva artificial. Rev. Odontol. Univ. Cid. São Paulo 2018 jul/set 30(3) 246-55

ISSN 1983-5183

Tabela 2. Média e desvio-padrão dos valores de acidez titulável* de diferentes sabores de balas duras, após diluição em água (G-1) e saliva artificial (G-2).

\begin{tabular}{llll}
\hline \hline Sabores & Marca comercial & G-1 (água) & G-2 (saliva artificial) \\
\hline Cereja & Mini Halls $^{\oplus}$ & $200,0 \pm 0 \mathrm{eA}$ & $0,0 \pm 0,0 \mathrm{cB}$ \\
Extraforte & Mini Halls $^{\circledast}$ & $1366,7 \pm 57,74 \mathrm{dA}$ & $200,0 \pm 0,0 \mathrm{bB}$ \\
Mentol & Mini Halls $^{\oplus}$ & $1666,7 \pm 57,74 \mathrm{cA}$ & $333,3 \pm 115,47 \mathrm{bB}$ \\
Melancia & Mini Halls $^{\oplus}$ & $3833,3 \pm 208,70 \mathrm{bA}$ & $600,0 \pm 0,0 \mathrm{aB}$ \\
Mentol & Valda $^{\oplus}$ & $200,0 \pm 0,0 \mathrm{eA}$ & $0,0 \pm 0,0 \mathrm{cB}$ \\
Limão & Melagrião $^{\oplus}$ & $5066,7 \pm 152,75 \mathrm{aA}$ & $300,0 \pm 0,0 \mathrm{bB}$ \\
\hline \hline
\end{tabular}

Médias seguidas da mesma letra minúscula na vertical e maiúscula na horizontal não diferem significativamente entre si pelo teste de Tukey $(\mathrm{p}<0,05)$. Volume $(\mu \mathrm{L})$ de $\mathrm{NaOH} 0,1 \mathrm{M}$ para alcançar $\mathrm{pH} 7,0$.

\section{DISCUSSÃO}

Nas últimas décadas, fatores como o aumento da prosperidade, disponibilidade e propaganda de novos produtos ácidos industrializados contribuíram para o aumento do risco à erosão dental em crianças e adolescentes ${ }^{17,18}$. Observou-se também um aumento da procura por produtos sem açúcar na composição, muitas vezes rotulados como sendo seguros para os dentes. Produtos rotulados com os termos "livre de açúcar" ou "zero açúcar" podem gerar uma falsa segurança, pois são adquiridos com o propósito de não causarem danos à estrutura dentária, por não serem cariogênicos. Entretanto, podem conter ácidos na sua composição e representar um fator de risco para a erosão dental $1^{10}$.

Na maioria dos produtos analisados foi identificada segundo o fabricante a presença do ácido cítrico. $O$ tipo de ácido influencia no potencial erosivo de um produto. $O$ ácido cítrico, presente em muitos produtos industrializados, apresenta um comportamento mais complexo perante os tecidos mineralizados. Numa solução aquosa ele se apresenta de 3 distintas formas: íons $\mathrm{H}^{+}$, ânions ácidos (citrato) e moléculas de ácido não dissociado, com as concentrações desses produtos variando de acordo com o pH e a constante de dissociação ácida ${ }^{19}$. O produto citrato apresenta ação quelante complexando-se com os íons cálcio presentes nos tecidos dentários duros e na saliva, prolongando o desgaste erosivo sofrido pela estrutura dentária ${ }^{19}$. Outro agravante é que sua concentração tende a se elevar com o aumento do $\mathrm{pH}$, promovendo a erosão dental em faixas de $\mathrm{pH}$ entre 4-6 ${ }^{12}$.

A presença de ácidos nos produtos analisados reflete diretamente no seu pH e acidez titulável, propriedades relevantes para a cinética da erosão dental, uma vez que a quantidade de dissolução dos cristais de apatita aumenta à medida que o $\mathrm{pH}$ diminui ${ }^{4,12}$.

Nesta pesquisa, os valores de $\mathrm{pH}$ das balas e pastilhas diluídas em água variaram entre 2,8 (limão Melagrião) e 6,1 (Mentol Valda). A maioria apresentou valores de pH inferiores a 5,5, corroborando com diversos estudos $16,20,21,22,23,24,25,26$. Quando diluídas em saliva artificial, todas elevaram seu pH para valores superiores a 5,5, de tal forma que, ao se comparar os grupos G-1 e G-2, observaram-se diferenças significativas. A elevação do $\mathrm{pH}$ após diluição na saliva artificial pode ser justificada pelos efeitos de diluição e tamponamento exercidos pela saliva artificial. Esse comportamento das soluções ácidas foi observado em outros estudos ${ }^{16,22,24}$.

$\mathrm{O}$ pH é uma propriedade importante para compreender a dinâmica da erosão dental. Conceitua-se como $\mathrm{pH}$ crítico o pH no qual a solução é saturada em relação aos produtos de solubilidade do mineral dental, 
RITA MR, FARIAS MMAG, SILVEIRA EG. Potencial erosivo de pastilhas e balas duras "zero açúcar" dissolvidas em água e saliva artificial. Rev. Odontol. Univ. Cid. São Paulo 2018 jul/set 30(3) 246-55

ISSN 1983-5183

como o esmalte. Quando o pH da solução está inferior ao crítico, diz-se que está subsaturada e, portanto, capaz de provocar desmineralização dos tecidos dentários duros. Por outro lado, quando o pH está acima do crítico, cria-se condições para a remineralização, ou seja, crescimento dos cristais de apatita ${ }^{27}$. Porém, o pH crítico também depende da concentração de cálcio, fosfato e em menor intensidade de flúor, pois esses íons interferem no grau de saturação da solução, que direcionará para a perda ou ganho mineral4,12,28. Assim, a subsaturação da solução é uma precondição para a desmineralização e a supersaturação necessária para o crescimento do cristal ${ }^{12}$.

O pH crítico de 5,5 usado para a dinâmica da cárie dental foi por muitos anos também utilizado para a erosão dental. Recentemente questiona-se este valor e infere-se que não há pH crítico fixo para a erosão do esmalte dental, uma vez que este dependerá da presença de minerais na solução, especialmente cálcio. Soluções ácidas com cálcio na sua composição são consideradas supersaturadas em relação ao produto de solubilidade do esmalte, não provocando o desgaste erosivo. Entretanto, também foi observado que soluções com valores de pH inferiores a 3,9 são sempre subsaturadas e, portanto, potencialmente erosivas, independente da presença de cálcio na solução, que poderia agir apenas como modulador da velocidade de dissolução ${ }^{28}$. No presente estudo pode-se considerar a maioria das soluções dissolvidas em água como potencialmente erosivas por apresentarem valores de $\mathrm{pH}$ inferiores a 3,9.

A acidez titulável refere-se à concentração total de todos os tipos de ácidos que se dissociam para fornecer íons $\mathrm{H}+$ entre valores de $\mathrm{pH}$ inicial e final'12. Quando ácidos orgânicos estão presentes em uma solução como a saliva, parte se encontra na forma não dissociada e parte dissociada. A acidez titulável está diretamente relacionada com a concentração de ácido não dissociado presente numa solução e, por se tratar de uma molécula não ionizada, tem a capacidade de se difundir mais facilmente através do esmalte abaixo da superfície, onde ocorre sua dissociação, liberando íons H+, criando uma condição local de subsaturação que favorece a desmineralização ${ }^{29}$. Esse comportamento do ácido não dissociado permite a formação de uma camada subsuperficial presente nas lesões de erosão que atualmente é denominada de "near-surface demineralization". Dessa forma, as lesões de erosão em esmalte não são exclusivamente superficiais, mas também contemplam uma pequena camada subsuperficial ${ }^{29}$.

Nossos resultados demonstraram que após a diluição em água (G-1) a acidez titulável ( $\mu \mathrm{L}$ de $\mathrm{NaoH}$ 0,1M) variou entre 5066,7 (limão Melagrlão ${ }^{\circledR}$ ) e 200 (mentol Valda ${ }^{\circledR}$ e cereja Mini Halls ${ }^{\circledR}$ ). Destacamos o perfil dos sabores melancia Mini Halls ${ }^{\circledR}$ e limão Melagrião ${ }^{\circledR}$ que apresentaram maior acidez titulável em ambos os grupos (G-1 e G-2). Esse comportamento sugere que tais produtos apresentam uma maior concentração de ácido não dissociado.

Na comparação entre os grupos observou-se redução da acidez titulável após diluição na saliva artificial (G-2) em todos os sabores, demonstratando a capacidade de tamponamento exercida pela saliva artificial. Achado semelhante ao de estudos anteriores ${ }^{22,24}$.

Elevada acidez titulável aumenta o tempo que a saliva necessita para neutralizar o ácido, aumentando seu potencial desmineralizante ${ }^{27}$. Assim, produtos podem apresentar potenciais erosivos distintos mesmo apresentando $\mathrm{pH}$ semelhante. Estudo recente demonstrou que a acidez titulável de uma bebida influencia mais o $\mathrm{pH}$ salivar depois do consumo de uma bebida ácida do que o $\mathrm{pH}$ da bebida ${ }^{30}$.

Compreende-se que a erosão dental é modulada por fatores químicos, comportamentais, biológicos, entre outros. A saliva representa o mais importante fator biológico na proteção dos tecidos dentários duros contra a erosão dental. Sua ação protetora inicia-se a partir do momento em que o estímulo ácido provoca 
ISSN 1983-5183

o aumento do fluxo salivar, criando um ambiente que favorece a atuação dos seus mecanismos de limpeza, capacidade tampão, influxo de minerais. Além disso, a película adquirida formada a partir de proteínas salivares age como uma membrana semipermeável que previne o contato do ácido com a superfície dentária. Dessa forma, pela atuação do conjunto dessas propriedades a saliva interfere no risco individual à erosão dental|13,15. Pacientes com hipossalivação decorrentes de doenças e medicações estão mais expostos à erosão. Nesses indivíduos, mesmo produtos com baixa erosividade podem representar um fator de risco.

Foi demonstrado pela literatura que após o consumo de balas duras há aumento do fluxo e queda do $\mathrm{pH}$ salivar. Seu retorno a normalidade ocorrerá em torno de 2 a 5 minutos após cessado o consumo ${ }^{21,31,}$ 32. Embora o retorno à neutralidade tenda a ocorrer num curto espaço de tempo, as balas e pastilhas duras, como as utilizadas neste estudo, tendem a ser dissolvidas lentamente na saliva, prolongando seu contato com os tecidos dentários ${ }^{31,32}$. Estudos comprovam que, embora durante o consumo de balas ácidas a saliva exerça um efeito protetor, este é parcial ${ }^{16,32}$.

Este modelo de estudo apresenta limitação, uma vez que um ambiente in vivo engloba todo o dinamismo da cavidade bucal. A saliva artificial utilizada neste estudo apresenta composição iônica e sistemas tampões semelhantes à saliva natural, porém na cavidade bucal os indivíduos podem apresentar fluxo variável, o que refletirá diretamente no seu potencial protetor, além das variações de composição, ou seja, da qualidade da saliva entre indivíduos. Dessa forma, estudos in vivo e in situ são importantes para melhor compreensão do papel da saliva na dinâmica da erosão dental, bem como para quantificar o desgaste erosivo nos tecidos dentários provocado por balas ácidas.

Os alimentos ácidos industrializados têm papel importante na etiologia da erosão dental; assim, devese retardar tanto quanto possível a exposição de crianças a produtos industrializados ácidos, no intuito de reduzir o risco à erosão dental ${ }^{17}$.

\section{CONCLUSÃO}

De acordo com os resultados, a maioria das balas e pastilhas analisadas são ácidas. A diluição em saliva artificial elevou o pH e reduziu a acidez titulável, reduzindo consequentemente o potencial erosivo desses produtos.

\section{AGRADECIMENTOS}

Ao Programa de Iniciação Científica Artigo 170/Governo do Estado de Santa Catarina/Pró-Reitoria de Pesquisa, Pós-Graduação, Extensão e Cultura da Universidade do Vale do Itajaí - UNIVALI, que financiou a pesquisa. 
RITA MR, FARIAS MMAG, SILVEIRA EG. Potencial erosivo de pastilhas e balas duras "zero açúcar" dissolvidas em água e saliva artificial. Rev. Odontol. Univ. Cid. São Paulo 2018 jul/set 30(3) 246-55

ISSN 1983-5183

\section{REFERÊNCIAS}

1. CATELAN A, Guedes APA, Santos PH. Erosão dental e suas implicações sobre a saúde bucal. RFO 2010 jan.-abr.;15(1):83-6.

2. VASCONCELOS FMN, Vieira SCM, Colares V. Dental erosion: diagnosis, prevention and management under oral health. Rev bras ciênc saúde 2010 14(1):59-64.

3. THOMAS MS, Vivekananda Pai AR, Yadav A. Medication-related dental erosion: a review. Compendium of continuing education in dentistry (Jamesburg, NJ : 1995) 2015 Oct;36(9):662-6; quiz 8.

4. WEST NX, Joiner A. Enamel mineral loss. Journal of dentistry 2014 Jun;42 Suppl 1(S211.

5. NAHAS Pires Correa MS, Nahas Pires Correa F, Nahas Pires Correa JP, Murakami C, Mendes FM. Prevalence and associated factors of dental erosion in children and adolescents of a private dental practice. International journal of paediatric dentistry 2011 Nov;21(6):451-8.

6. FARIAS MMAG, Silveira EG, Schmitt BHE, Araújo SM, Baier IBA. Prevalência da erosão dental em crianças e adolescentes brasileiros. Salusvita 2013 32(2):18798.

7. SALAS MM, Nascimento GG, Vargas-Ferreira F, Tarquinio SB, Huysmans MC, Demarco FF. Diet influenced tooth erosion prevalence in children and adolescents: Results of a meta-analysis and meta-regression. Journal of dentistry 2015 Aug;43(8):865-75.

8. SOVIK JB, Skudutyte-Rysstad R, Tveit AB, Sandvik L, Mulic A. Sour sweets and acidic beverage consumption are risk indicators for dental erosion. Caries research 2015 49(3):243-50.

9. MOYNIHAN PJ. The role of diet and nutrition in the etiology and prevention of oral diseases. Bull World Health Organ 2005 Sep.;83(9):694-9.

10. NADIMI H, Wesamaa H, Janket SJ, Bollu P, Meurman JH. Are sugar-free confections really beneficial for dental health? British dental journal 2011 Oct 7;211(7):E15.

11. FURTADO JR, Freire VC, Messias DCF, Turssi CP. Aspectos físico-químicos relacionados ao potencial erosivo de bebidas ácidas. RFO, Passo Fundo 2010 set./ dez. ;15(3):325-30.

12. SHELLIS RP, Featherstone JD, Lussi A. Understanding the chemistry of dental erosion. Monographs in oral science 2014 25(163-79.

13. BUZALAF MA, Hannas AR, Kato MT. Saliva and dental erosion. Journal of applied oral science : revista FOB 2012 Sep-Oct;20(5):493-502.

14. MAGALHAES AC, Wiegand A, Rios D, Honorio HM, Buzalaf MA. Insights into preventive measures for dental erosion. Journal of applied oral science : revista FOB 2009 Mar-Apr;17(2):75-86. 
RITA MR, FARIAS MMAG, SILVEIRA EG. Potencial erosivo de pastilhas e balas duras "zero açúcar" dissolvidas em água e saliva artificial. Rev. Odontol. Univ. Cid. São Paulo 2018 jul/set 30(3) 246-55

ISSN 1983-5183

15. HARA AT, Zero DT. The potential of saliva in protecting against dental erosion. Monographs in oral science 2014 25(197-205.

16. WAGONER SN, Marshall TA, Qian F, Wefel JS. In vitro enamel erosion associated with commercially available original-flavor and sour versions of candies. Journal of the American Dental Association (1939) 2009 Jul;140(7):906-13.

17. GAMBON DL, Brand HS, Veerman EC. Dental erosion in the 21st century: what is happening to nutritional habits and lifestyle in our society? British dental journal 2012 Jul 27;213(2):55-7.

18. STEWART KF, Fairchild RM, Jones RJ, Hunter L, Harris C, Morgan MZ. Children's understandings and motivations surrounding novelty sweets: a qualitative study. International journal of paediatric dentistry 2013 Nov;23(6):424-34.

19. FEATHERSTONE JD, Lussi A. Understanding the chemistry of dental erosion. Monographs in oral science 2006 20(66-76.

20. BRAND HS, Gambon DL, Paap A, Bulthuis MS, Veerman EC, Amerongen AV. The erosive potential of lollipops. International dental journal 2009 Dec;59(6):358-62.

21. BRAND HS, Gambon DL, Van Dop LF, Van Liere LE, Veerman EC. The erosive potential of jawbreakers, a type of hard candy. International journal of dental hygiene 2010 Nov;8(4):308-12.

22. BONVINI B, Soraes AK, Farias MMAG, Araújo SM, Schmitt BEH. Mensuração do potencial erosivo de balas dissolvidas em água e saliva artificial. Rev Odontol UNESP 2016 45(3):154-8.

23. DAVIES R, Hunter L, Loyn T, Rees J. Sour sweets: a new type of erosive challenge? British dental journal 2008 Jan 26;204(2):E3; discussion 84-5.

24. FARIAS MMAG, Oliveira MML, Schmitt BHE, Silveira EG, Araújo SM. Erosive potential of sugar-free hard candies dissolved in water and artificial saliva. Braz $J$ Oral Sci 2016 15(1):4.

25. LAZZARIS M, Farias MMAG, Araújo SM, Schmitt BHE, Silveira EG. Erosive potential of commercially available candies. Pesqui Bras Odontopediatria Clín Integr 2015 15(1):7-12.

26. SILVA JG, Farias MMAG, Silveira EG, Araújo SM, Schmitt BHE. Evaluation of the erosive potential of acidic candies consumed by children and tennagers. J Pharmacy Nutrition Sciences 2013 3(4):262-5.

27. LUSSI A, Schlueter N, Rakhmatullina E, Ganss C. Dental erosion--an overview with emphasis on chemical and histopathological aspects. Caries research 2011 45 Suppl 1(2-12.

28. LUSSI A, Carvalho TS. Erosive tooth wear: a multifactorial condition of growing concern and increasing knowledge. Monographs in oral science 2014 25(1-15. 
ISSN 1983-5183

29. SHELLIS RP, Barbour ME, Jesani A, Lussi A. Effects of buffering properties and undissociated acid concentration on dissolution of dental enamel in relation to $\mathrm{pH}$ and acid type. Caries research 2013 47(6):601-11.

30. GAMBON DL, Brand HS, Nieuw Amerongen A. [Acidic candies affect saliva secretion rates and oral fluid acidity]. Nederlands tijdschrift voor tandheelkunde 2007 Aug;114(8):330-4.

31. TENUTA LM, Fernandez CE, Brandao AC, Cury JA. Titratable acidity of beverages influences salivary pH recovery. Brazilian oral research 2015 29(

32. JENSDOTTIR T, Nauntofte B, Buchwald C, Bardow A. Effects of sucking acidic candy on whole-mouth saliva composition. Caries research 2005 Nov-Dec;39(6):468-74.

RECEBIDO EM 24/11/2017

ACEITO EM 28/09/2018 